\title{
PERCEPÇÃO DO FAMILIAR CUIDADOR SOBRE A INDEPENDENCIA FUNCIONAL, - NIVEL COGNITIVO E O ESTADO EMOCIONAL DO IDOSO
}

\author{
Paulo Frassinetti D. Nascimento ${ }^{1}$ \\ llary Gondim Dias Sousa² \\ Maria Fernanda Lopes ${ }^{3}$ \\ Nilza Moura Marques ${ }^{4}$ \\ Allan Pablo Lameira ${ }^{5}$
}

resumo

O envelhecimento é um processo natural, caracterizado por mudanças físicas, psicológicas e sociais individualizadas que podem resultar no quadro de dependência funcional. Nesse contexto, faz-se necessário

1 Bacharel em Enfermagem. Mestrando do Programa de Pós-Graduação em Neurociência Cognitiva e Comportamento da Universidade Federal da Paraiba (UFPB). E-mail: paulonasc94@gmail.com.

2 Graduanda do curso de Medicina da Universidade Federal de Campina Grande (UFCG). E-mail: ilary.gondim@gmail.com.

3 Graduanda do curso de Medicina da Universidade Federal de Campina Grande (UFCG). E-mail: mariafernandalopes5@gmail.com.

4 Graduanda do curso de Medicina da Universidade Federal de Campina Grande (UFCG). E-mail: nilzamarquesm@gmail.com.

5 Bacharel em Fisioterapia. Mestre em Neuroimunologia. Doutor em Neurociências. Professor da disciplina de Anatomia Humana (UFCG). E-mail: allanpablolameira@gmail.com. 
o auxílio de um cuidador, que geralmente é representado por algum membro familiar. $O$ presente estudo é uma pesquisa experimental, transversal, de caráter quantitativo, que objetivou compreender como o idoso é percebido (avaliado). Foi realizado o questionamento do familiar cuidador sobre o estado geral do idoso, averiguando se a sua avaliação condiz com o resultado de escalas já validadas e que abordam os seguintes aspectos: a independência funcional, o nível cognitivo e o estado emocional. Os dados foram analisados por meio do teste de correlação de Spearman com as variáveis: percepção do acompanhante (PA); nível cognitivo (NC); independência funcional (IF); e estado emocional (EE). A análise resultou em correlação significativa apenas entre as variáveis: PA e NC; IF e NC. O fato de ter ocorrido apenas dois resultados significativos sugere que o familiar cuidador apresenta dificuldades em captar/identificar qual o estado geral do idoso que está sobre os seus cuidados. Ou seja, possivelmente, questões como escolaridade, idade avançada, condições socioeconômicas, entre outros fatores intrínsecos do indíviduo podem determinar a capacidade perceptiva e empática, acarretando na redução da qualidade assistencial oferecida ao idoso dependente.

\section{palavras-chave}

Idoso. Familiar Cuidador. Incapacidade Funcional. Envelhecimento Cognitivo. Depressão.

\section{Introdução}

O processo de envelhecimento, também conhecido como senescência, trata-se uma etapa da vida caracterizada por mudanças físicas, psicológicas e sociais que acometem de forma única cada pessoa. Nesta fase, a saúde destaca-se por ser um dos aspectos mais afetados. Levando-se em consideração o aumento da longevidade da população idosa, vários estudos são desenvolvidos com o objetivo de melhorar a qualidade de vida na terceira idade.

O conceito de qualidade de vida envolve diversas esferas, como o nível sociocultural, a faixa etária, a capacidade funcional, o ambiente em que está inserido, bem como suas aspirações pessoais (FECHINE; TROMPIERI, 2012; TAVARES et al., 2017). Dentre os aspectos que possuem impacto direto na qualidade de vida do idoso, destacam-se a independência funcional, o nível cognitivo e o estado emocional, fatores que necessitam estar em equilíbrio com 
o envelhecimento psíquico e biológico (SANTOS; CUNHA, 2013; TAVARES et al., 2017).

O idoso que mantém sua independência funcional (capacidade de exercer sua autonomia), deve ser considerado saudável, ainda que apresente uma ou mais doenças crônicas (VERAS, 2009, 2016). Durante a senescência surgem alterações orgânicas típicas, como perda de peso, redução da massa corpórea, mobilidade intestinal e da força muscular, além da diminuição na acuidade visual e da função vestibular (RIBEIRO; COSENZA, 2013), alterações que resultam no aumento do risco de quedas e fraturas (FREITAS, 2014; CANÊDO; LOURENÇO, 2017). Assim, identificar precocemente os idosos com risco para incapacidade funcional possibilita o planejamento de intervenções com vistas à potencialização da autonomia e redução da dependência (BARBOSA et al., 2014).

Todavia, alterações a nível cognitivo durante o processo de envelhecimento acarretam muitas vezes em consequências mais lesivas, fazendo-se necessário o acompanhamento minucioso do comportamento do idoso, com o objetivo de identificar indícios da redução da capacidade cognitiva (SILVEIRA; PORTUGUEZ, 2017). O declínio gradual ou súbito, geralmente está relacionado com a inteligência fluida, com a memória de trabalho, memória episódica, raciocínio e orientação espacial, funções que interferem diretamente na capacidade funcional e resultam na perda da autonomia (BUCZYLOWSKA; PETERMANN, 2016).

Além das citadas anteriormente, a senescência é passível de alterações que provocam ansiedade, medo, insegurança, conflitos e sentimentos de solidão. $\mathrm{O}$ aparecimento de doenças, a perda de vínculos afetivos e a exclusão social constituem fatores importantes para a vulnerabilidade a transtornos mentais, como a depressão (FECHINE; TROMPIERI, 2012; MELO et al., 2017). A depressão é um transtorno mental que envolve tanto fatores biológicos como psicossociais, estando associado ao elevado risco de morbidade e mortalidade (NÓBREGA et al., 2015). Apesar disso, não se dá a devida atenção ao desenvolvimento de depressão em idosos. Em geral, os profissionais da saúde percebem os sintomas depressivos como manifestações decorrentes do processo de envelhecimento, fato esse que não necessariamente seja algo atrelado a essa fase da vida (LIMA et al., 2016).

Portanto, a perda ou redução da capacidade funcional devido a alterações físicas, cognitivas ou emocionais ocasionam graves limitações na autonomia, levando ao impedimento da realização das atividades cotidianas (PINTO JUNIOR et al., 2016; TAVARES et al., 2017).

Neste contexto, segundo o Estatuto do Idoso (BRASIL, 2004) é dever e responsabilidade do Estado, da sociedade e da família, assegurar à pessoa idosa, $\mathrm{o}$ 
acesso aos direitos sociais básicos já elencados no art. $6^{0}$ da Constituição Federal de 1988 (BRASIL, 2016), os quais consistem em direito a saúde, alimentação, educação, cultura, liberdade, dignidade, respeito, entre outros. Todavia, em alguns casos, faz-se necessário a presença de um cuidador na vida deste idoso dependente. Geralmente, o cuidado é delegado a algum membro do núcleo familiar que irá assumir as funções de cuidador (PEREIRA; SOARES, 2015).

Cabe destacar que cuidar de um idoso é uma atividade difícil e que necessita de dedicação quase sempre integral. O grande problema é que geralmente devido a fatores como: baixa escolaridade, estresse causado pela sobrecarga física e emocional, falta de experiência, idade avançada ou mesmo falta de empatia; torna-se difícil oferecer um suporte adequado (MAIA et al., 2018).

Desta forma, o presente estudo possui o objetivo de compreender como o estado geral do idoso é percebido/avaliado pelo membro familiar responsável por seus cuidados e qual o impacto dessa avaliação na qualidade de vida daquele. Para este fim, comparamos a opinião do familiar cuidador sobre o estado geral do ente cuidado, com os resultados de escalas já validadas que mensuraram aspectos relacionados com a qualidade de vida (independência funcional para realização das atividades diárias, nível cognitivo e estado emocional).

A relevância acadêmica, científica e social deste trabalho surge devido à carência na literatura sobre o tema, bem como à possibilidade de se obter avanços no cuidado de idosos dependentes funcionalmente. Além de possibilitar tais avanços em relação a este, o presente trabalho também possibilita avaliar os possíveis fatores que podem interferir na qualidade do cuidado oferecido pelo familiar cuidador. Por exemplo, o não acompanhamento do estado físico e psicológico do familiar cuidador, bem como a falta de capacitação e suporte por parte dos serviços públicos, potencializam as possibilidades de expor os idosos a situações de risco. Assim, o acompanhamento e suporte adequado para o familiar cuidador, possibilitará que este forneça para a equipe de saúde, informações fundamentais sobre a situação do idoso sobre sua responsabilidade.

\section{Metodologia}

Trata-se de uma pesquisa experimental, transversal e de caráter quantitativo, conduzida de acordo com a Declaração de Helsinque de 1975, aprovada pelo Comitê de Ética em Pesquisa da Universidade Federal de Campina Grande, sob o Protocolo CAAE: 45144215.3.0000.5575. 
Participaram da pesquisa quarenta voluntários (vinte idosos e seus respectivos acompanhantes) selecionados aleatoriamente, adotando-se os seguintes critérios de exclusão: a) idoso com menos de 65 anos; b) idosos acompanhados pelo cônjuge também idosos; c) idosos com doenças terminais, com demência grave e incapacidade de comunicação; d) recusar-se em assinar o termo de consentimento livre e esclarecido.

Para a coleta de dados, foram utilizados os seguintes intrumentos: a) questionário para dados sociodemográficos dos idosos (idade, sexo, escolaridade, ocupação e uso de medicamentos); b) Mini Exame de Estado Mental (MEEM); c) Escala Barthel (EB); d) Escala de Depressão Abreviada de Zung (EDAZ).

O MEEM avalia as funções cognitivas, sendo composto por questões agrupadas de acordo com a função avaliada: orientação temporal e espacial, registro de memória de fixação, atenção e cálculo, memória de evocação, linguagem e praxia construtiva. A pontuação total varia entre 0 e 30 pontos, e após ajustes segundo escolaridade, como preconizado por "Sugestões para o uso do Mini Exame do Estado Mental no Brasil", o ponto de corte foi de 20 para analfabetos e 25 para alfabetizados (SANTOS; PAVARINI, 2010).

Já a EB, avalia o nível de independência do sujeito para a realização de dez atividades básicas de vida: comer, higiene pessoal, uso do sanitário, tomar banho, vestir e despir, controle dos esfíncteres, deambular, transferência da cadeira para a cama, subir e descer escadas. O EB varia de 0 a 100 pontos, sendo que zero, significa dependência total para todas as atividades de vida diária (AVDs) e cem, independência total. Segundo Sulter e colaboradores (1999), o ponto de corte estabelecido entre dependência e independência é de 60 pontos, onde os indivíduos com 85 pontos ou mais são considerados habitualmente independentes, necessitando apenas de uma assistência mínima.

Por fim, a versão abreviada da Escala de Depressão de Zung, validada por Díaz e colaboradores (2005), avaliou o estado emocional dos idosos. Ela apresenta apenas 10 perguntas e cada item da escala apresenta quatro alternativas pontuadas de 1 a 4 pontos. Do total de 40 pontos possíveis, consideram-se os escores iguais ou menores que 22 pontos como ausência de depressão, de 23 a 35 como depressão moderada e acima de 35, depressão grave.

A realização do estudo ocorreu no domicílio do idoso e/ou familiar cuidador. A pesquisa foi iniciada com uma pergunta feita ao familiar, sem a presença do idoso: "Como você acha que o idoso está: ruim, regular, bom ou ótimo?". Pontuações foram diretamente associadas as respostas: ruim - 0; regular - 1; bom - 2 e ótimo - 3. Em seguida, sem a presença do familiar cuidador, foram aplicados os questionários com o idoso. A sequência de aplicação dos 
instrumentos foi aleatória e o tempo de aplicação dependia do estado geral do entrevistado.

Após coleta, os dados foram tabulados no software Microsoft Office Excel 2007 e analizados através do software Statistica 7.0 da Statsoft para plataforma Windows. Devido possuirem características de dados não paramétricos (não intervalados e mensurados apenas no nível ordinal), os dados foram submetidos a estatística inferencial por meio do coeficiente de correlação de Sperman, assumindo-se a probabilidade de erro tipo $\alpha$ de $5 \%(p<0,05)$, para verificar a existência e intensidade de possíveis correlações entre as seguintes variáveis: percepção do acompanhante (PA); nível cognitivo (NC); independência funcional (IF) e estado emocional (EE). Cabe destacar que a váriavel "percepção do acompanhante (PA)", refere-se a como o cuidador familiar avalia o estado (geral) idoso sob sua responsabilidade, indo desde ascpetos físicos a emocionais.

\section{Resultados}

Os dados sociodemográficos demonstram que dos 20 idosos participantes da pesquisa, $04(20 \%)$ eram homens, com média de idade de 76,75 anos $( \pm 5,9)$ e $16(80 \%)$ eram mulheres, com média de idade de 73,62 anos $( \pm 5,6)$. Dentre todos, $45 \%$ eram analfabetos, $45 \%$ apresentavam o primeiro grau incompleto e $10 \%$ possuíam o nível superior completo.

Após análise, os resultados revelaram correlação significativa entre duas situações específicas. A primeira ocorreu entre a variável "percepção do familiar cuidador" e "nível cognitivo" (@ Sperman=0,65; p>.001), mostrando que quanto maior a pontuação atribuída ao idoso pelo cuidador, maior o nível cognitivo daquele pelo MEEM (Gráfico 1). 
Gráfico 1 - Correlação positiva entre percepção do acompanhante (PA) e nível cognitivo do idoso

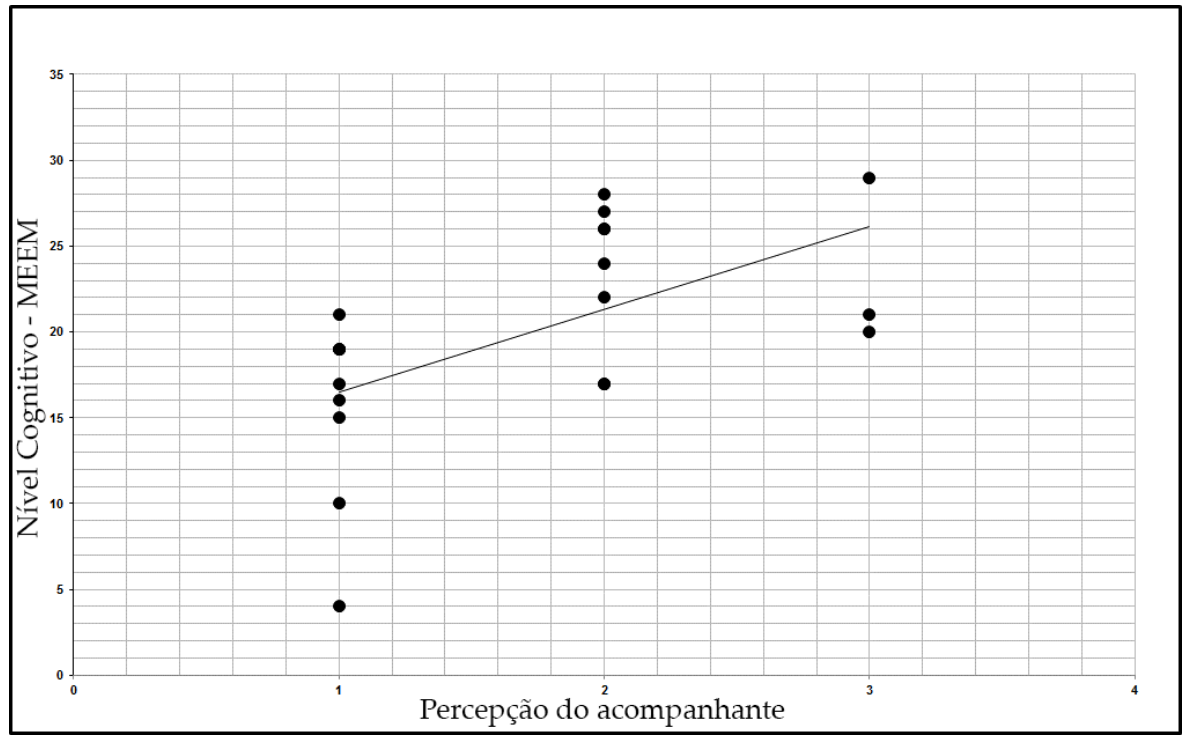

Fonte: Elaborado pelos autores, 2019.

A segunda correlação ocorreu entre as variáveis "independência funcional e nível cognitivo" (@ Sperman=0,45; p>.046), revelando que quanto maior a independência funcional do idoso, avaliada pela EB (scores mais altos), maior o nível cognitivo desse idoso, segundo o MEEM (Gráfico 2). Nenhuma outra correlação significativa entre as demais variáveis foi encontrada (Tabela 1). 


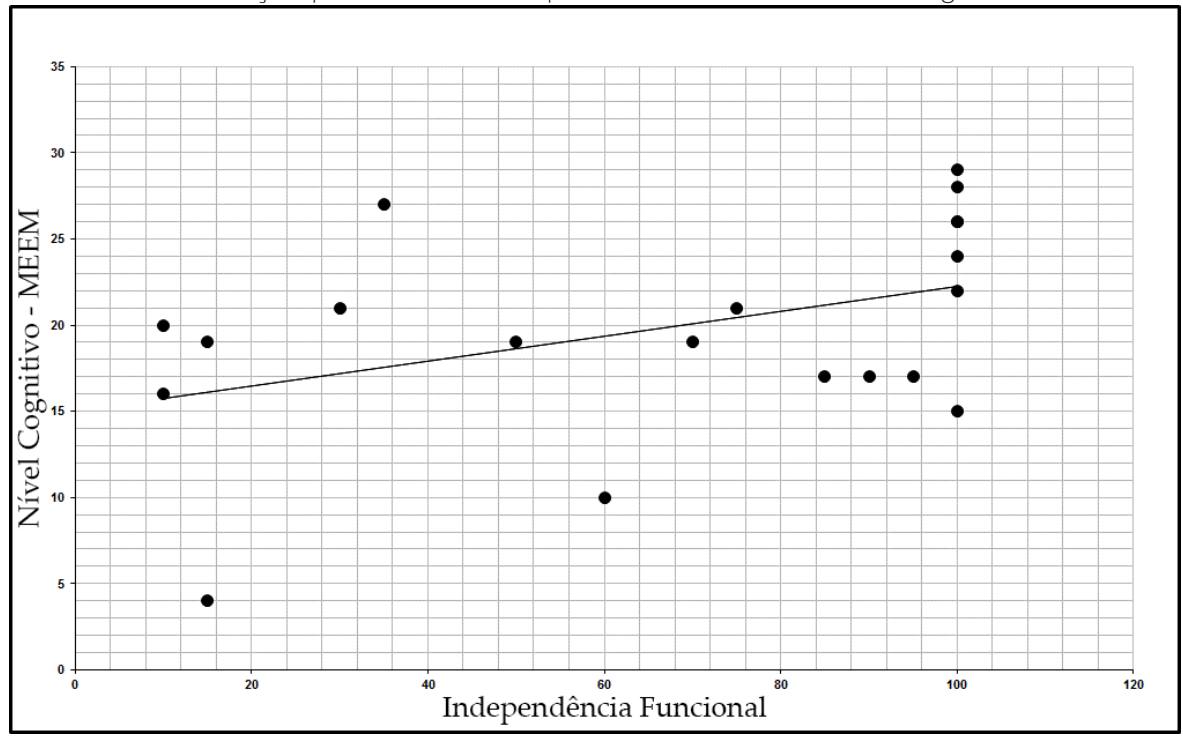

Fonte: Elaborado pelos autores, 2019.

Tabela 1 - Correlação entre as variáveis

\begin{tabular}{lllll}
\hline & Amostra & $\rho$ Spearman & $\mathbf{t}(\mathbf{N}-2)$ & Significância $(\mathbf{p})$ \\
\hline PA \& IF & 20 & 0,370821 & 1,69404 & 0,107491 \\
PA \& EE & 20 & $-0,266947$ & $-1,17521$ & 0,255224 \\
PA \& NC & 20 & 0,653256 & 3,66054 & 0,001789 \\
FF \& EE & 20 & 0,107780 & 0,45995 & 0,651059 \\
IF \& NC & 20 & 0,450695 & 2,14202 & 0,046118 \\
EE \& NC & 20 & $-0,025787$ & $-0,10944$ & 0,914064 \\
\hline
\end{tabular}

Legenda: PA = Percepção do Acompanhante; IF = Independência Funcional; $\mathrm{EE}=$ Estado Emocional; NC = Nível Cognitivo.

Fonte: Elaborada pelos autores, 2019. 
O envelhecimento é um processo dinâmico e progressivo, resultante de alterações funcionais, bioquímicas, morfológicas e/ou psicológicas que culminam na perda progressiva da capacidade de adaptação do indivíduo ao meio ambiente, tornando-o vulnerável à maior incidência de processos patológicos (BERTAZONE et al., 2016; CERTO et al., 2016).

Todavia, cabe salientar que embora seja uma fase da vida mais propensa ao desenvolvimento de enfermidades, a senescência não pode ser compreendida como sinônimo de "doença". Inúmeros fatores são determinantes para o surgimento de agravos a saúde do indivíduo. Entre eles estão incluídos a predisposição genética, fatores socioeconômicos, educacionais, culturais e a existência de políticas públicas que atuem efetivamente para o envelhecimento ativo (saudável) (MINAYO; COIMBRA JR., 2002; PEEL et al., 2005; LEITE et al., 2017).

Entretanto, a incapacidade funcional e a diminuição da autonomia são os principais problemas que podem afetar os idosos, segundo Carmo, Oliveira e Morelato (2016). A realização das atividades de vida diária, quando limitadas ou impossibilitadas de serem realizadas, pode levar os idosos a depender de outras pessoas. Essa dependência pode ser ocasionada principalmente por enfermidades físicas, por doenças mentais ou ambas as condições (SANTOS; CUNHA, 2013). De acordo com Ramos (2003), durante a senescência, o fator determinante para o envelhecimento saudável é o nível de autonomia. $\mathrm{Ou}$ seja, a capacidade de gerir sua própria vida, pois independente da presença de doenças crônicas como diabetes e hipertensão (desde que controláveis), o indivíduo pode ser visto como um idoso saudável.

Aqui, o presente estudo buscou avaliar como o estado geral do idoso é percebido (avaliado) pelo familiar cuidador, verificando se quem vive próximo ao idoso o identifica e entende o seu estado físico e mental/emocional. A pesquisa desenvolveu-se através da comparação da avaliação feita pelo familiar cuidador sobre o estado geral do idoso e o resultado de três escalas validadas que quantificam diferentes aspectos da saúde do mesmo (independência funcional, nível cognitivo e estado emocional).

Nossos resultados revelaram que o familiar cuidador foi capaz de avaliar (perceber) o nível cognitivo do idoso de forma correspondente a avaliação (mensuração) realizada pelo MEEM, indicando que quanto melhor é a avaliação do idoso pelo cuidador, melhor é o seu estado cognitivo. Esse resultado corrobora com os achados de Guedes e colaboradores (2013) que realizaram um estudo para comparar as avaliações efetuadas por idosos (autorrelato) e por seus cuidadores (heterorrelato) quanto à qualidade de vida. Guedes e 
colaboradores observaram que o nível cognitivo do idoso era bem avaliado pelos cuidadores e que não havia diferenças entre as avaliações realizadas pelo cuidador ou pelo idoso em relação a sua qualidade de vida.

Porém, a ausência de correlação significativa entre a percepção do familiar cuidador e a independência funcional do idoso, evidencia que esse familiar possui dificuldades em avaliar a capacidade física de execução das atividades de vida diária pelo idoso. Essa dificuldade de avaliação das capacidades funcionais pode fazer que o familiar cuidador exponha inadvertidamente o idoso a riscos desnecessários. Por exemplo, a falta de conhecimento sobre a capacidade de realizar diversas atividades cotidianas (subir escada ou tomar banho) pode aumentar o risco de quedas. É importante ressaltar que nosso estudo utiliza uma única escala de aferição da independência funcional (Escala Barthel).

Além disso, também foi observada a ausência de correlação significativa entre a percepção do familiar cuidador e o estado emocional do idoso, sugerindo que por algum motivo, o estado emocional do idoso não foi detectado e/ou assimilado pelo cuidador. Segundo Ramos e colaboradores (2019), a desesperança, a sensação de inutilidade, alterações de sono e déficit de lazer são os principais sintomas relacionados a estados depressivos em idosos. O tratamento do idoso depressivo está baseado em ações de caráter medicamentoso, psicoterápico e de mudanças do padrão de vida. Porém, é extremamente necessário que o familiar cuidador saiba identificar essas alterações emocionais para que possa solicitar e/ou encaminhar o idoso a um serviço de tratamento adequado.

Entretanto, é dever e responsabilidade do Estado atuar para que o idoso, dependente ou não, possua uma qualidade de vida satisfatória, seja diretamente por meio de profissionais capacitados ou através da capacitação do cuidador familiar. Para isso, políticas públicas voltadas para o idoso são cruciais no atendimento, como o Estatuto do Idoso (BRASIL, 2004) e a Política Nacional de Saúde do Idoso (PNSI), criada através da Portaria nº 1395/1999, do Ministério da Saúde (MS) (BRASIL, 2006), que além de visar à promoção do envelhecimento saudável, prevenção de doenças, preservação e reabilitação da capacidade funcional, atua na introdução de meios que facilitem a prevenção de danos, sejam eles físicos ou mentais (VERAS; OLIVEIRA, 2018).

Outro resultado encontrado foi uma correlação significativa entre o nível de independência funcional e o nível cognitivo do idoso. Esse resultado mostra que idosos com maior independência funcional apresentam melhor preservação da capacidade cognitiva. Talmelli e colaboradores (2013), investigando idosos com Alzheimer, também observaram essa correlação entre a capacidade funcional do idoso e seu estado cognitivo. Porém, nossos resultados não mostraram correlação entre a independência funcional e o estado 
emocional e nem entre o estado emocional e o nível cognitivo. Essas ausências de correlação sugerem que o estado emocional do idoso não depende da sua capacidade funcional e nem do nível cognitivo. Ou seja, o idoso poderia ser independente funcionalmente e ter um bom nível cognitivo, mas desenvolver o quadro de depressão.

\section{Conclusão}

Os resultados aqui discutidos sugerem que, em comparação com as escalas citadas anteriomente, o familiar cuidador possuiu dificuldades em captar qual o estado geral do idoso que está sobre os seus cuidados. Possivelmente, questões como escolaridade, idade avançada, condições socioeconômicas, entre outros fatores intrínsecos do indíviduo, podem ser determinantes para a capacidade perceptiva e empática, acarretando na redução da qualidade assistencial oferecida ao idoso dependente (GUTIERREZ; FERNANDES; MASCARENHAS, 2017).

Mesmo a qualidade de vida dos idosos sendo algo multidimensional que envolve aspectos funcionais, cognitivos e emocionais, existem poucos estudos que abordem a percepção e compreensão dessa tríade pelo familiar cuidador, bem como a atuação do Estado através do Mínistério da Saúde, na orientação, capacitação e acompanhamento do responsável por tais cuidados com idosos dependentes. Portanto, faz-se necessário a realização de mais estudos que enfoquem sobre o papel e a importância do familiar cuidador. Além disso, buscar identificar quais fatores interferem na capacidade avaliativa do estado geral do idoso, como também a sua capacitação, visando a detecção precoce de possíveis afecções, é crucial para que seja possível reduzir o risco de expor o idoso e a família a situações ainda mais debilitantes.

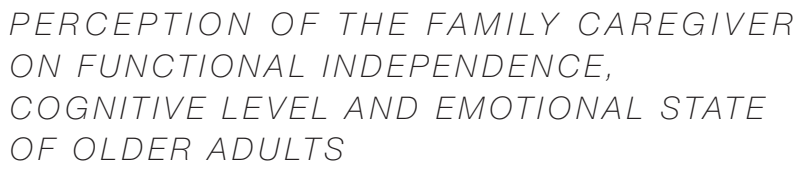

abstract

Aging is a natural process, characterized by individualized physical, psychological and social changes that can result in functional dependency. In this context, the help of a caregiver is necessary, and is usually represented by a family member. The present study is an 

to understand how older adults are perceived (evaluated). Family caregivers were questioned about the general condition of the older adults, checking whether their assessment is consistent with the result of scales already validated. The following aspects were addressed: functional independence, cognitive level and emotional state. Data were analyzed using Spearman's correlation test with the variables: companion's perception (PA); cognitive level (NC); functional independence (IF) and emotional state (EE). The analysis resulted in a significant correlation only between the variables: PA and NC; IF and NC. The fact that there were only two significant results suggests that the family caregiver has difficulties in capturing/identifying the general state of the older adults who are under their care. Issues such as education, advanced age, socioeconomic conditions, among other intrinsic factors of the individual, can possibly determine the perceptive and empathic capacity, resulting in a reduction in the quality of care offered to the dependent older adults.

keywords

Older Adults. Caregiver. Functional Disability. Cognitive Aging. Depression.

\author{
referências
}

\begin{abstract}
BARBOSA, Bruno Rossi et al. Avaliação da capacidade funcional dos idosos e fatores associados à incapacidade. Ciência \& Saúde Coletiva, Rio de Janeiro, v. 19, n. 8, p. 3317-3325, 2014. Disponivel em: http://www.scielo.br/scielo.php?script=sci_ arttext\&pid=S1413-81232014000803317\&lng=en\&nrm=iso. Acesso em: jul. 2016.
\end{abstract}

BERTAZONE, Thaís Mara Alexandre et al. Multidisciplinary/interdisciplinary actions in the care of elderly with Alzheimer's Disease. Revista da Rede de Enfermagem do Nordeste, Fortaleza, v. 17, n. 1, p. 144-153, 2016.

BRASIL. Constituição da República Federativa de 1988. Brasília, DF: Presidência da República, 2016. Disponível em: http://www.planalto.gov.br/ccivil_03/constituicao/ constituicao.htm. Acesso em: 8 out. 2019.

BRASIL. Estatuto do idoso: lei federal no 10.741, de 01 de outubro de 2003. Brasília, DF: Secretaria Especial dos Direitos Humanos, 2004

BRASIL. Ministério da Saúde. Portaria n².528, de 19 de outubro de 2006. Aprova a Política Nacional de Saúde da Pessoa Idosa. Brasília, DF: Ministério da Saúde, 2006. Disponível em: https://bvsms.saude.gov.br/bvs/saudelegis/gm/2006/prt2528 _19_10_2006.html. Acesso em: 8 out. 2019.

BUCZYLOWSKA, Dorota; PETERMANN, Franz. Age-related differences and heterogeneity in executive functions: analysis of NAB executive functions module scores. Archives of Clinical Neuropsychology, Oxford, v. 31, n. 3, p. 254-262, 2016. 
CANÊDO, Ana Cristina; LOURENÇO, Roberto A. Determinantes do envelhecimento bem-sucedido. Revista do Hospital Universitário Pedro Ernesto, Rio de Janeiro, v. 16, n. 1, p. 51-55, 2017.

CARMO, Júlia Fabres do; OLIVEIRA, Elizabete Regina Araújo; MORELATO, Renato Lirio. Functional disability and associated factors in elderly stroke survivors in Vitória, Brazil. Revista Brasileira de Geriatria e Gerontologia, Rio de Janeiro, v. 19, n. 5 , p. 809-818, out. 2016.

CERTO, Ana et al. A síndrome da fragilidade nos idosos: revisão da literatura. Actas de Gerontologia, Porto, v. 2, n. 1, p. 1-11, 2016.

DÍAZ, Luis Alfonso et al. Propuesta de una versión abreviada de la escala de Zung para depresión. Colombia Médica, Colombia, v. 36, n. 3, 2005.

FECHINE, Basílio Rommel Almeida; TROMPIERI, Nicolino. O processo de envelhecimento: as principais alterações que acontecem com o idoso com o passar dos anos. InterSciencePlace, Campos dos Goytacazes, v. 1, n. 20, p. 106-132, 2012.

FREITAS, Mónica Correia de. Efeito da idade e do género sobre os afetos em idosos: mediação da capacidade motora percebida ou da competência funcional percebida? Dissertação (Mestrado em Psicologia) - Escola de Ciências Sociais, Universidade de Évora, Évora, 2014

GUTIERREZ, Lucila Ludmila Paula; FERNANDES, Neisa Rejane Machado; MASCARENHAS, Marcello. Caracterização de cuidadores de idosos da região metropolitana de Porto Alegre (RS): perfil do cuidado. Saúde em Debate, Rio de Janeiro, v. 41, n. 114, p. 885-898, 2017.

LEITE, Bruna Silva et al. A vulnerabilidade dos cuidadores de idosos com demência: estudo descritivo transversal. Revista Brasileira de Enfermagem, Brasília, DF, v. 70, n. 4, p. 682-688, 2017

LIMA, Ana Maraysa Peixoto et al. Depressão em idosos: uma revisão sistemática da literatura. Revista de Epidemiologia e Controle de Infecção, Santa Cruz do Sul, v. 6, n. 2, 2016. Disponível em: https://online.unisc.br/seer/index.php/epidemiologia/article/ view/6427. Acesso em: 25 fev. 2020

MAIA, Juliana Moreira et al. Perfil dos cuidadores de idosos de uma instituição de longa permanência de Imperatriz - MA. Revista Ciência em Extensão, São Paulo, v. 14, n. 2, p. 43-54, 2018

MELO, Beatriz Rodrigues de Souza et al. Cognitive and functional assessment about elderly people users of health public service. Escola Anna Nery, Rio de Janeiro, v. 21, n. 4, e20160388, 2017.

MINAYO, Maria Cecília de Souza; COIMBRA JR., Carlos E. A. (org.). Antropologia, saúde e envelhecimento. Rio de Janeiro: Editora FIOCRUZ, 2002.

NÓBREGA, Isabelle Rayanne Alves Pimentel da et al. Fatores associados à depressão em idosos institucionalizados: revisão integrativa. Saúde em Debate, Rio de Janeiro, v. 39, n. 105, p. 536-550, 2015.

PEEL, Jennifer L. et al. Ambient air pollution and respiratory emergency department visits. Epidemiology, Cambridge, MA, v. 16, n. 2, p. 164-174, 2005.

PEREIRA, Lírica Salluz Mattos; SOARES, Sônia Maria. Fatores que influenciam a qualidade de vida do cuidador familiar do idoso com demência. Ciência \& Saúde Coletiva, Rio de Janeiro, v. 20, n. 12, p. 3839-3851, 2015.

PINTO JUNIOR, Elzo Pereira et al. Dependência funcional e fatores associados em idosos corresidentes. Cadernos Saúde Coletiva, Rio de Janeiro, v. 24, n. 4, p. 404-412, 2016

RAMOS, Fabiana Pinheiro et al. Fatores associados à depressão em idoso. Revista Eletrônica Acervo Saúde, Ouro Fino, v. supl. 19, e239, 2019. 
RAMOS, Luiz Roberto. Fatores determinantes do envelhecimento saudável em idosos residentes em centro urbano: Projeto Epidoso, São Paulo. Cadernos de Saúde Pública, Rio de Janeiro, v. 19, n. 3, p. 793-797, 2003

RIBEIRO, Angela Maria; COSENZA, Ramon M. Envelhecimento normal do sistema nervoso. In: MALLOY-DINIZ, Leandro F.; FUENTES, Daniel; COSENZA, Ramon M. (org.). Neuropsicologia do envelhecimento: uma abordagem multidimensional. Porto Alegre: Artmed, 2013. p. 78-99.

SANTOS, Ariene Angelini dos; PAVARINI, Sofia Cristina lost. Perfil dos cuidadores de idosos com alterações cognitivas em diferentes contextos de vulnerabilidade social. Revista Gaúcha de Enfermagem, Porto Alegre, v. 31, n. 1, p. 115-122, 2010. Disponível em: https://www.scielo.br/scielo.php?script=sci_arttext\&pid=\$1983-14472010000100016. Acesso em: fev. 2017

SANTOS, Gerson Souza; CUNHA, Isabel Cristina Kowal Olm. Avaliação da capacidade funcional de idosos para o desempenho das atividades instrumentais da vida diária: um estudo na atenção básica à saúde. Revista de Enfermagem do Centro Oeste Mineiro, Divinópolis, v. 3, n. 3, p. 820-828, 2013.

SILVEIRA, Michele Marinho da; PORTUGUEZ, Mirna Wetters. Analysis of life quality and prevalence of cognitive impairment, anxiety, and depressive symptoms in older adults. Estudos de Psicologia, Campinas, v. 34, n. 2, p. 261-268, 2017.

TALMELLI, Luana Flávia da Silva et al. Doença de Alzheimer: declínio funcional e estágio da demência. Acta Paulista de Enfermagem, São Paulo, v. 26, n. 3, p. 219-225, 2013.

TAVARES, Renata Evangelista et al. Envelhecimento saudável na perspectiva de idosos: uma revisão integrativa. Revista Brasileira de Geriatria e Gerontologia, Rio de Janeiro, v. 20, n. 6, p. $878-889,2017$.

VERAS, Renato. Care pathway for the elderly: detailing the model. Revista Brasileira de Geriatria e Gerontologia, Rio de Janeiro, v. 19, n. 6, p. 887-905, 2016. Disponível em: https://doi.org/10.1590/1981-22562016019.160205. Acesso em: 10 out. 2019

VERAS, Renato. Envelhecimento populacional contemporâneo: demandas, desafios e inovações. Revista de Saúde Pública, São Paulo, v. 43, n. 3, p. 548-554, 2009.

VERAS, Renato Peixoto; OLIVEIRA, Martha. Envelhecer no Brasil: a construção de um modelo de cuidado. Ciência \& Saúde Coletiva, Rio de Janeiro, v. 23, n. 6, p. 19291936, 2018. Disponível em: https://doi.org/10.1590/1413-81232018236.04722018. Acesso em: 7 out. 2019.

Data de Submissão: 01/07/2019

Data de Aprovação: 29/02/2020 